\title{
AOR
}

Selected Papers of \#AolR2020:

The $21^{\text {st }}$ Annual Conference of the

Association of Internet Researchers

Virtual Event / 27-31 October 2020

\section{FACELESS YOUTUBERS: HOW CONTENT CREATORS SHAPE AUDIENCE EXPECTATIONS}

Ian Dunham

Kennesaw State University

"Okay, (calming zen gesture) let's do this. This is so weird. Welcome back to my channel...this is my face." - fastASMR

On August 18, 2018, the YouTube channel fastASMR revealed her face to her audience of several hundred thousand with little fanfare. Prior to this moment, she had only ever shown the bottom half of her face, concealing her nose and eyes. She is not alone in being cautious about disclosing her face, and belongs to a surprisingly large group of channels this paper is calling Faceless YouTubers. As a platform that users value for its ability to provide 'a critique, a goal, a community, and a context' (Jenkins quoting Alex Juhasz, 2009, p. 125), this specific subset of video creators employs unique practices related to surveillance, disclosure and information flow.

YouTube has achieved ubiquity as the de facto video platform for amateur content. Its maturation as a platform can be seen through a collection of social norms, audience expectations, and politics, all of which weave themselves into the technosocial fabric of digital life. As such, certain practices that regulate the flow of information have become normalized across a certain subset of YouTube channels. This paper will look at this practice among YouTubers that are "faceless" - content creators who do not reveal their faces on camera while voluntarily revealing many other aspects of their identity. Specifically, a content analysis is performed on these channels: The Lockpicking Lawyer, AvE, SteadyCraftin, This Old Tony, bigclivedotcom, CGP Grey, and fastASMR. The wide range of categories these channels belong to (DIY, self-help, science and technology, home crafting, electrical appliance teardown, and audio therapy) indicates that choosing to remain faceless is not a function of a certain type of material, but rather a salient trend that crosscuts YouTube genres.

Anonymity has been explored since the internet granted such communicative affordances (Scott, 1998). It has a strong correlation with the online disinhibition effect, Suggested Citation (APA): Dunham, I. (2020, October). Faceless Youtubers: How Content Creators Shape Audience Expectations. Paper presented at AolR 2020: The $21^{\text {th }}$ Annual Conference of the Association of Internet Researchers. Virtual Event: AolR. Retrieved from http://spir.aoir.org. 
a phenomenon in which users more readily volunteer information "more boldly than they would in a face-to-face situation" (Clark-Gordon et al., 2019). Furthermore, invisibility (a state reached through self-selecting which information is shown to outside users) has been shown to interact with anonymity to encourage the online disinhibition effect, both in toxic and prosocial instances. While anonymity deals with being completely unidentifiable, invisibility - which includes preventing access to "gender, weight, age, occupation, ethnic origin and residential location" (Lapidot-Lefler \& Barak, 2015)proves a key concept since Faceless YouTubers generate a bond with their audience through this selective disclosure. In a medium that is mostly visual, remaining faceless is both a protest against cultural expectations within the format and an effort to shift focus to other audiovisual artifacts that empower these creators, such as shots of power tools and workspaces, animated content, a YouTuber's hands, and even sounds (see Figure 1). It should be noted that all the analyzed channels contain content with the YouTuber as its central focus, rather than other types of faceless material that might focus on narrative, news reporting, or documentary material. In this way, their representation is achieved through a novel method that does not require facial exposure.

YouTubers (and many other types of internet users for that matter) have always been forced to mitigate trolling, an unfortunate practice that is, ironically, afforded through anonymity (Burgess \& Green, 2018, p. 80). Because YouTube has been classified as an engine that can fuel hatred and bigotry just as often as it can propagate learning and creativity (Tufekci, 2018), YouTubers constantly experiment with strategies to create meaningful content. In fact, trolling is one of the major hurdles YouTubers must overcome: "Learning how to 'manage' trolls, both practically and emotionally, is one of the core competencies required for effective or enjoyable participation" (Burgess \& Green, 2018, p. 80). The Faceless YouTubers in this study manage trolls in two important ways: by refusing to reveal their faces, their appearance cannot be the fodder of trolling. Simultaneously, though, by placing an emphasis on other aspects of their identity (their job, their hobbies, the physical space they inhabit), any trolling can only be aimed at what viewers hear and see (see Figure 1). 


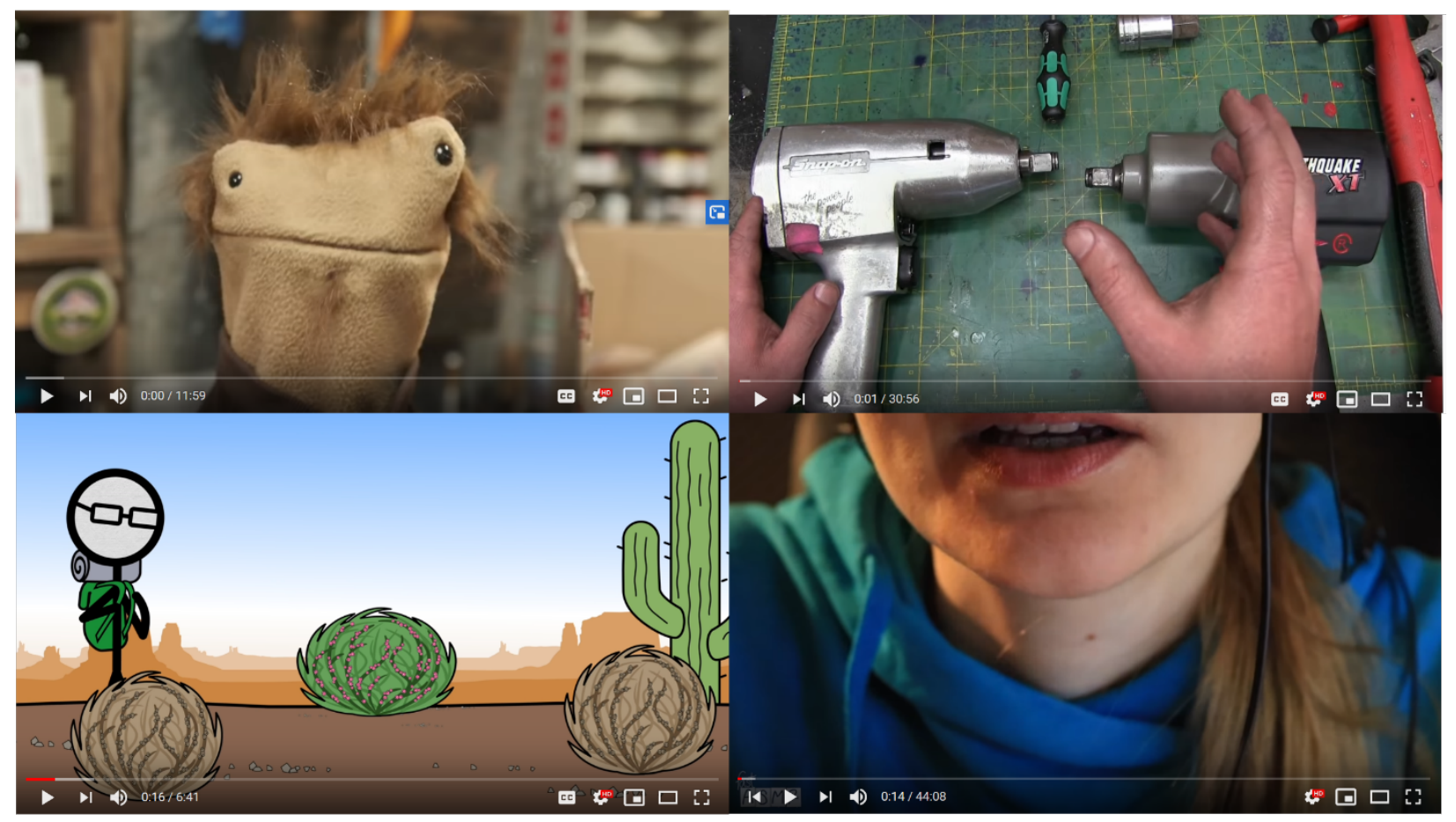

Figure 1: Faceless YouTubers' Identity (Clockwise from top left: SteadyCraftin, AvE, CGP Grey, and fastASMR)

The content analysis of these Faceless YouTubers reveals a variety of motivations, but several have to do with identity construction. In the case of SteadyCraftin, his face is never in frame, and his hands are always covered by gloves, a choice most certainly made to conceal his race (his voice is always unconcealed, and his accent is raceambiguous, perhaps a choice to further conceal his identity). Simultaneously, though, Steadycraftin, has shared deeply personal information, such as the fact that he suffers from PTSD and other psychological problems, as well as the fact that he has a wife and children. Similarly, AvE and This Old Tony have both shown their children's faces on camera. These examples suggest that YouTubers feel empowered through remaining faceless, possibly making them feel more at ease and thereby allowing for increased disclosure about the topic at hand (Clark-Gordon et al., 2019). They also demonstrate that Faceless YouTubers selectively share information depending on a range of motivations, all of which revolve around imparting meaningful content to specific audiences while maintaining privacy.

While Faceless YouTubers exercise unique strategies for controlling the flow of information to audiences, they are certainly not alone in developing methods of control. Transgender YouTubers, for instance, have demonstrated equally compelling methods for maintaining privacy, sharing relevant information, establishing a sense of identity, and cultivating a supportive community (Raun, 2016). Similarly, anorexic YouTubers have propagated a sense of agency through sharing stories of recovery that deal with narratives of physical bodies (Holmes, 2016). While overlap exists in the strategies these communities employ and those of the Faceless YouTubers I have analyzed, the specific configuration of approaches at hand are distinct as they "shape social norms and reflexively negotiate the ethics of online behavior, operating from a position of grounded, insider knowledge" (Burgess \& Green, 2018, p. 80). These strategies, 
discussed only briefly here because of space constraints, will be expanded upon in the presentation.

\section{References}

Burgess, J. \& Green, J. (2009). YouTube: Online video and participatory culture. Cambridge: Polity.

Burgess, J. \& Green, J. (2018). YouTube: Online video and participatory culture. Cambridge: Polity.

Clark-Gordon, C. Bowman, N. Goodboy, A. Wright, A. (2019) Anonymity and Online Self-Disclosure: A Meta-Analysis. Communication Reports, 32:2, 98-111

Holmes, S. (2016).'My anorexia story': girls constructing narratives of identity on YouTube. Cultural Studies, 31(1), 1-23.

Lapidot-Lefler, N., \& Barak, A. (2015). The benign online disinhibition effect: Could situational factors induce self-disclosure and prosocial behaviors?. Cyberpsychology: Journal of Psychosocial Research on Cyberspace, 9(2).

Scott, C. R. (1998). To reveal or not to reveal: A theoretical model of anonymous communication. Communication Theory, 8, 381-407.

Raun, T. (2016). Out online: Trans self-representation and community building on YouTube. New York: Routledge.

Tufekci, Z. (2018, March 11). YouTube: the Great Radicalizer. New York Times, p. SR6. 